\title{
NOVO SISTEMA PARA GESTÃO DE EVENTO BASEADO EM BANCO \\ DE DADOS COM INTERFACE WEB PARA A SECRETARIA DA ESCOLA DE ENGENHARIA DA UFF
}

\author{
Bruna de Mello Almeida - brmello@id.uff.br \\ Alexandre Santos de laVega-alexandresantoslavega@id.uff.br \\ Grupo PET-Tele - http://www.telecom.uff.br/pet \\ Universidade Federal Fluminense - UFF \\ Escola de Engenharia - TCE \\ Departamento de Engenharia de Telecomunicações - TET \\ Rua Passo da Pátria, 156 / Bloco D / Sala 504 \\ 24.210-240 - Niterói - Rio de Janeiro
}

Resumo: Este trabalho apresenta um novo sistema para o projeto de colaboração realizado pelo grupo PET-Tele para a Secretaria da Escola de Engenharia, da Universidade Federal Fluminense (UFF). O PET-Tele desenvolveu e implantou a primeira versão de um Sistema de Gestão para o evento SEMENGE 2018 (Semana de Engenharia), baseado em Banco de Dados, usando a linguagem SQL (Structured Query Language), com interface Web, empregando as linguagens HTML (HyperText Markup Language), PHP (Hypertext Processor) e CSS (Cascading Style Sheets). A motivação básica do projeto foi atender a uma demanda emergencial da Secretaria, que notificou a perda do sistema anterior. O sistema proposto pelo PET-Tele entrou em operação a tempo de atender ao evento e foi utilizado com sucesso. Para a versão 2019 do evento, agora denominado de SEMENDE, o PET-Tele desenvolveu um novo sistema, o qual é descrito nesse documento.

Palavras-chave: Programa de Educação Tutorial (PET). Sistema de Gestão. Evento acadêmico. Banco de Dados. Acesso via Web.

\section{INTRODUÇÃO}

O Programa de Educação Tutorial (PET) (MINISTÉRIO DA EDUCAÇÃO, 2020) exige que os bolsistas dos seus grupos, ao serem submetidos a uma formação complementar, desenvolvam atividades que possuam, conjuntamente, itens relativos às áreas de Pesquisa, Ensino e Extensão, que consigam algum tipo de penetração no curso ao qual pertencem e que realizem trabalhos de cooperação com outros grupos, ligados ou não ao seu curso de origem. Logo, o PET busca atitudes inovadoras em educação.

Procurando atender aos requisitos do Programa, o grupo PET do Curso de Engenharia de Telecomunicações da Universidade Federal Fluminense (PET-Tele/UFF) (PET-TELE, 2020) tem realizado trabalhos na linha de banco de dados e interfaces Web.

A Secretaria da Escola de Engenharia, da Universidade Federal Fluminense (UFF), anualmente organizava o evento SEMENGE (Semana de Engenharia). Em 2018, a secretaria notificou que o sistema de gestão que era utilizado no evento foi perdido. Conhecendo o trabalho desenvolvido pelo PET-Tele, a secretaria convidou o grupo a desenvolver, em caráter emergencial, um novo sistema. O PET-Tele, aceitando o desafio, desenvolveu e implantou a primeira solução para um Sistema de Gestão, que foi usado no evento SEMENGE 2018 (RANGEL JR. et al., 2019). 
Em 2019, a Secretaria da Escola de Engenharia revalidou a demanda por um sistema de gestão para o evento, agora expandido e renomeado para SEMENDE (Semana de Engenharia e Design). O PET-Tele, novamente aceitando o desafio, desenvolveu e implantou uma nova solução para o Sistema de Gestão que foi usado no evento SEMENDE 2019.

O novo sistema desenvolvido é apresentado a seguir. A Seção 2 resume as motivações e os objetivos do projeto. As atividades realizadas são descritas na Seção 3. As Seções 4 e 5 descrevem, respectivamente, as versões atuais do Banco de Dados e da interface Web. Finalmente, as considerações finais são realizadas na Seção 6.

\section{MOTIVAÇÕES E OBJETIVOS}

Em iniciativas educacionais anteriores, procurando atender de uma forma unificada aos segmentos de pesquisa, de ensino e de extensão, o grupo PET-Tele desenvolveu atividades envolvendo banco de dados e interfaces $W e b$.

A Secretaria da Escola de Engenharia, da Universidade Federal Fluminense (UFF), organizava um evento anual denominado SEMENGE (Semana de Engenharia). O evento é constituído de inúmeras atividades, de diferentes tipos (palestra, mesa redonda, curso, minicurso, oficina). Ele recebe um público-alvo da ordem de 1000 pessoas. Para o controle do evento, diversos funcionários são alocados. Para o controle das atividades, alunos dos cursos de Engenharia são convidados a participar. Para dar suporte à gestão do evento, a Secretaria contava com um sistema computacional próprio, desenvolvido para esse fim. Porém, a quase totalidade do sistema de gestão que era utilizado no evento foi perdido, restando apenas a parte que atendia à pré-inscrição dos participantes. Sem um sistema computacional de apoio, todo o controle do evento deve ser realizado manualmente, com planilhas eletrônicas e/ou armazenamento de dados em papel, gerando redundância de informação, aumento de trabalho, aumento de erros, aumento do tempo empregado e redução de eficiência.

Conhecendo o trabalho desenvolvido pelo PET-Tele, a Secretaria convidou o grupo a desenvolver, em caráter emergencial, um novo sistema de suporte à gestão da SEMENGE.

Aceitando o desafio, o PET-Tele levantou as necessidades, realizou um grupo de estudos, desenvolveu e implantou a primeira solução para um novo Sistema de Gestão, que foi usado no evento SEMENGE 2018, com aprovação da Secretaria (RANGEL JR. et al., 2019).

Em 2019, a Escola de Engenharia expandiu o evento, incorporando o Curso de Desenho Industrial e renomeando-o para SEMENDE (Semana de Engenharia e Design). Mais uma vez, o PET-Tele foi convidado a desenvolver o Sistema de Gestão do evento. O grupo novamente aceitou o desafio e, cumprindo as mesmas etapas de projeto, desenvolveu e implantou uma nova solução para o Sistema de Gestão que foi usado no evento SEMENDE 2019.

O desenvolvimento do novo Sistema de Gestão para a SEMENDE é apresentado a seguir.

\section{DESENVOLVIMENTO DO NOVO SISTEMA DE GESTÃO}

O processo de desenvolvimento do novo Sistema de Gestão é descrito a seguir.

\subsection{Conhecimento acumulado pelo grupo}

O grupo PET-Tele possui experiência relativa a Bancos de Dados com acesso via Web. Isso foi atingido por meio de atividades internas do grupo e pelo desenvolvimento de projetos simples, colaborando com setores e/ou laboratórios da Escola de Engenharia (RANGEL JR. et al., 2019), (RANGEL JR. et al., 2017), (OLIVEIRA et al., 2016), (DINIZ et al., 2013) e (PET-TELE, 2020). 
Em grupos de estudos, com a colaboração do professor Carlos E. Pantoja (CEFET/RJ), foram trabalhados conceitos básicos sobre banco de dados e sua modelagem (YOUNG, 1983).

O PET-Tele possui experiência de projeto usando o Sistema de Gerenciamento de Banco de Dados (SGBD) MySQL, que utiliza SQL (Structured Query Language) como linguagem de programação e é um padrão de código aberto (MySQL, 2020) e (SQL, 2020).

Com a intenção de um acesso via rede, o grupo tem optado por uma interface de acesso $W e b$. Pensando em um aplicativo que possua facilidade de desenvolvimento, de instalação, de manutenção e de portabilidade, bem como seja de acesso simplificado, o grupo tem implementado interfaces baseadas em três linguagens de desenvolvimento Web: HTML (HyperText Markup Language), PHP (Hypertext Processor) e CSS (Cascading Style Sheets) (ACHOUR et al., 2020), (EIS, 2020), (LEWIS \& MOSCOVITZ, 2010), (NIEDERST, 2002), (PACIEVITCH, 2020), (PHP, 2020), (SILVA, 2020), (W3SCHOOLS, 2020).

\subsection{Sistema de gestão desenvolvido anteriormente}

A Secretaria da Escola de Engenharia organizava anualmente o evento SEMENGE (Semana de Engenharia) e contava com um sistema computacional especialmente desenvolvido para a gestão do evento. Porém, tal sistema foi perdido. Conhecendo o trabalho do PET-Tele, a Secretaria consultou o grupo sobre a possibilidade de desenvolver um novo sistema para a SEMENGE 2018. Embora houvesse pouco tempo para o desenvolvimento e a implantação, o grupo aceitou o desafio e um novo sistema foi implantado em uma rede interna da Secretaria, sendo utilizado na SEMENGE 2018 (RANGEL JR. et al., 2019). A Secretaria emitiu um Voto de Louvor pelo trabalho do PET-Tele, em Boletim de Serviço da UFF, indicando o sucesso do sistema implantado (Boletim de Serviço UFF 1, 2020).

\subsection{Novo sistema de gestão implantado}

Em 2019, a Escola de Engenharia decidiu expandir o evento SEMENGE. O Curso de Desenho Industrial foi incorporado ao evento, que foi renomeado para SEMENDE (Semana de Engenharia e Design).

Mais uma vez, o PET-Tele foi convidado a desenvolver o Sistema de Gestão do evento. O grupo novamente aceitou o desafio e, devido à expansão do evento, passou pelas mesmas etapas de projeto, desenvolveu e implantou uma nova versão para o Sistema de Gestão que foi usado no evento SEMENDE 2019.

Como primeiro passo, foram realizadas reuniões com funcionários da Secretaria, a fim de se levantar as novas especificações do problema. De posse das informações coletadas, abriram-se duas frentes. Por um lado, foi iniciada a elaboração de um modelo formal para a construção do Banco de Dados. De outro lado, foram feitas propostas iniciais para as interfaces $W e b$.

Novas funcionalidades foram implantadas para os participantes, tais como: (i) cadastro no sistema, via webpage; e (ii) acesso ao sistema (login) para obtenção de instruções para pagamento de inscrição, consulta à programação, cadastro em atividades, verificação de lista de espera e geração de certificado (se a base de dados acusar que o participante tem direito).

Para a administração do evento, o sistema continuou a atender às seguintes necessidades básicas: confirmação de pagamento da inscrição de participante, envio automático de confirmação da inscrição de participante, inserção e edição de dados relativos às atividades, geração de diversos tipos de relatórios. Com a intenção de incorporar, de uma maneira fácil, os relatórios do sistema em documentos da Secretaria, optou-se por gerá-los no formato CSV (Comma-Separated Values) (CSV-RFC, 2020) (CSV-WIKI, 2020). Por sua vez, os certificados seriam gerados no formato de um arquivo do tipo PDF (Portable Document Format) (PDF, 2020). 
O novo sistema foi implantado em uma rede interna da Secretaria e vários testes foram realizados, com funcionários que participariam do controle do evento. $\mathrm{O}$ sistema foi utilizado na SEMENDE 2019. A Secretaria emitiu um novo Voto de Louvor pelo trabalho do PETTele, em Boletim de Serviço da UFF, indicando o sucesso do novo sistema implantado (Boletim de Serviço UFF 2, 2020).

\section{VERSÃO ATUAL DO BANCO DE DADOS}

O Sistema de Gerenciamento de Banco de Dados (SGBD) utilizado foi o MySQL, que utiliza SQL (Structured Query Language) como linguagem de programação e é um padrão de código aberto (MySQL, 2020) e (SQL, 2020).

O banco associado ao Sistema de Gestão foi implementado em uma máquina principal, sendo acessado apenas pelas máquinas conectadas na rede interna da Secretaria.

Buscando a normalização dos dados e a redução de redundâncias, foram criadas sete tabelas, cinco principais (login, pagamento, usuario, atividade, presenca) e duas intermediárias (inscricao_ativ e atividade_dia), as quais armazenam e relacionam os dados das pessoas inscritas com os dados das atividades oferecidas.

As tabelas foram definidas segundo um padrão Entidade-Relacionamento (ER), com as seguintes relações entre elas: (i) login-usuario, com relação 1:1; (ii) pagamento-usuario, com relação 1:1; (iii) usuario-inscricao_ativ, com relação 1:n; (iv) inscricao_ativ-atividade, com relação n:1; (v) atividade_dia-atividade, com relação 1:1; (vi) atividade_dia-presenca, com relação 1:n; e (vii) presenca-usuario, com relação n:1.

As tabelas adotadas e suas conexões são apresentadas na Figura 1.

Figura 1 - Tabelas adotadas.

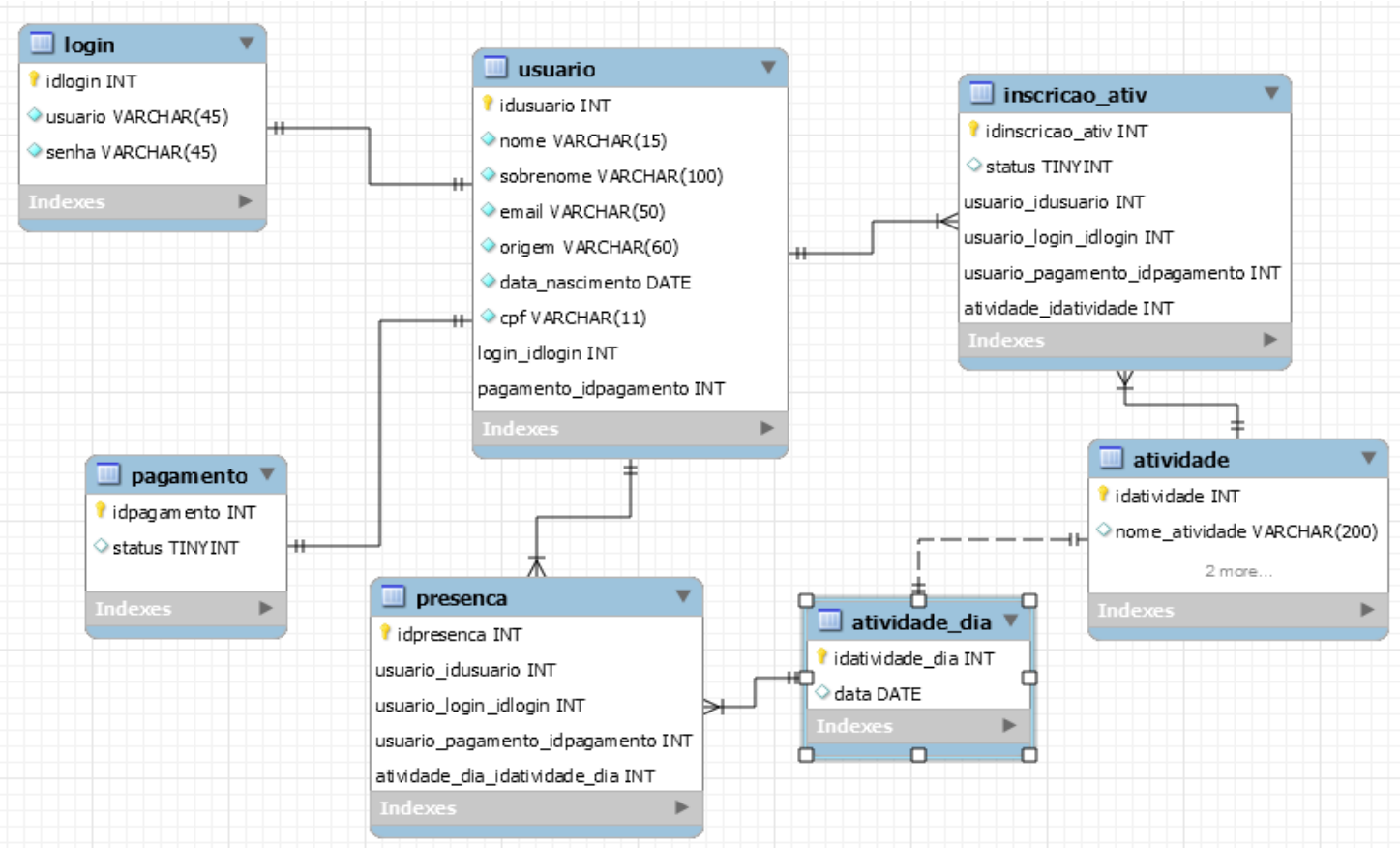




\section{VERSÃO ATUAL DA INTERFACE WEB}

Para acessar o banco de dados, o Sistema de Gestão conta com uma interface de acesso $W e b$, composta por um conjunto de webpages específicas para cada função do sistema. Para que ela possuísse facilidade de desenvolvimento, de instalação, de manutenção e de portabilidade, bem como fosse de acesso simplificado, o grupo decidiu manter uma interface baseada em três linguagens de desenvolvimento Web: HTML, PHP e CSS. A fim de permitir um acesso confortável em dispositivos móveis, as webpages possuem front-end responsivo.

Uma tela específica possibilita o cadastro dos participantes no evento. Os administradores são cadastrados diretamente no banco de dados. Para que os participantes e a administração tenham acesso ao sistema, é disponibilizada uma tela de acesso (login). Telas específicas reúnem as ações relativas ao participante e à administração. As funções disponíveis para os participantes são: inscrição em atividades, verificação de inscrições e suas listas de espera, verificação de pagamento, verificação de presenças e geração de certificados. $\mathrm{Na}$ inscrição em atividades, o participante é capaz de visualizar a lista de atividades e suas informações. Também é possível desistir de uma inscrição já efetuada. Na verificação de inscrições, o participante é capaz de checar a situação de sua inscrição, podendo receber os seguintes status: inscrito, aguardando pagamento ou em lista de espera. Caso encontre-se em lista de espera, é possível verificar em qual posição se encontra. Na verificação de pagamento, o participante pode obter instruções para o pagamento da taxa de inscrição no evento e também verificar o status de confirmação. Na verificação de presenças, é possível, durante o evento, que o participante verifique as suas presenças nas atividades em que está inscrito. Na geração de certificado, o participante que possua carga horária mínima em cada atividade inscrita consegue gerar um certificado contendo as atividades realizadas e a carga horária correspondente. As funções disponíveis aos administradores são: cadastramento de atividade, confirmação de pagamento, marcação de presenças e geração de relatórios. O cadastramento de atividades permite a inserção ou a retirada de atividades do banco, alterando de forma dinâmica o cronograma do evento. Pela confirmação de pagamento, é possível alterar o status de confirmação de pagamento de um participante. Em marcação de presenças, é possível marcar as presenças de cada participante em cada atividade. Na geração de relatórios, é possível gerar relatórios estatísticos (quantidade de participantes por atividade, taxa de presença por atividade, taxa de faltas) e relatórios de pagamentos para controle interno dos pagamentos recebidos pelos participantes.

Pela limitação de espaço, apenas algumas webpages são apresentadas nas Figuras 2 a 5.

Figura 2 - Tela de cadastro de participante.

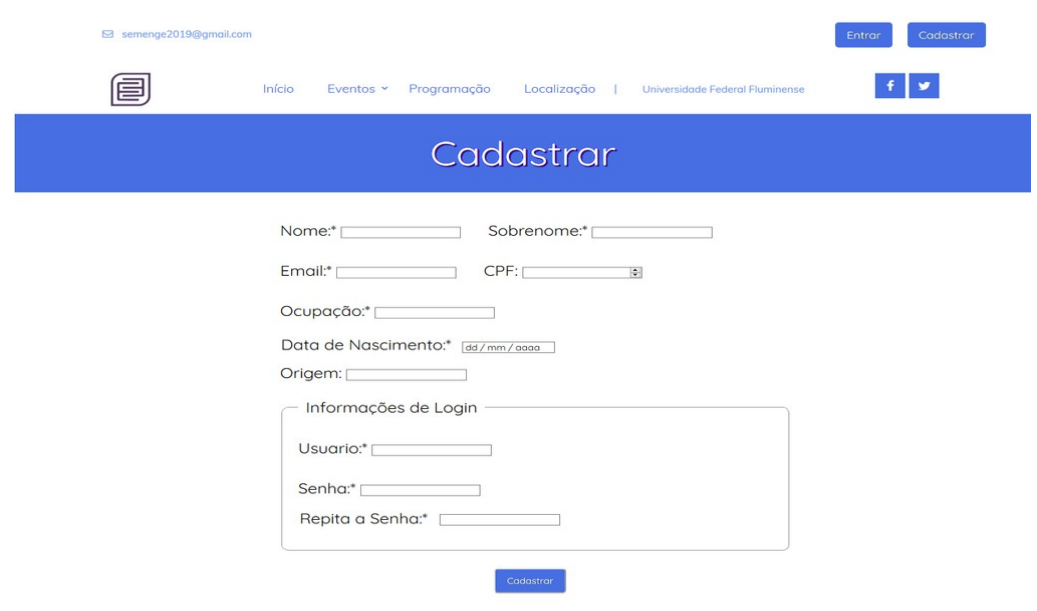


(C) COBENCE

"Os desafios para formar hoje o engenheiro do amanhã"
01 a 03 de dezembro Evento On-line

Figura 3 - Tela de acesso ao sistema (login).

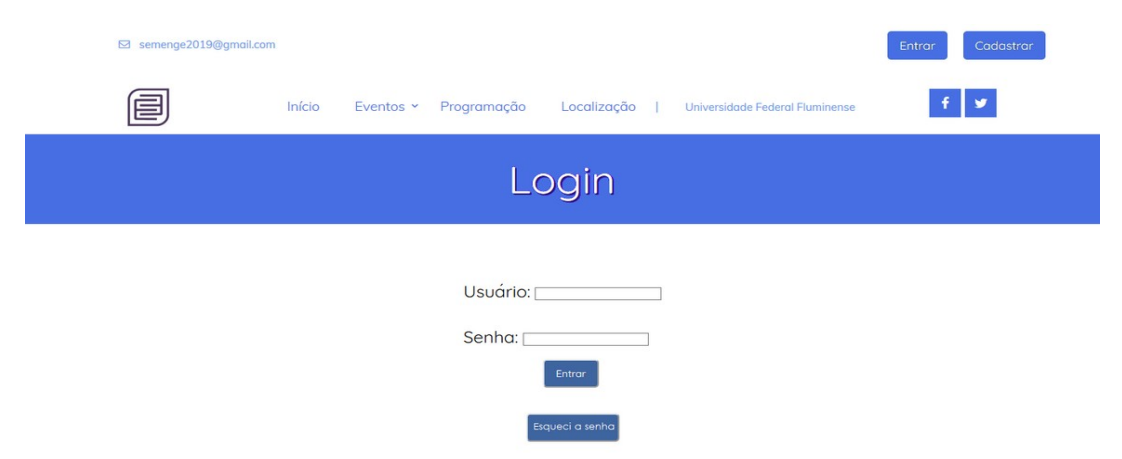

Figura 4 - Tela de ações de administração.

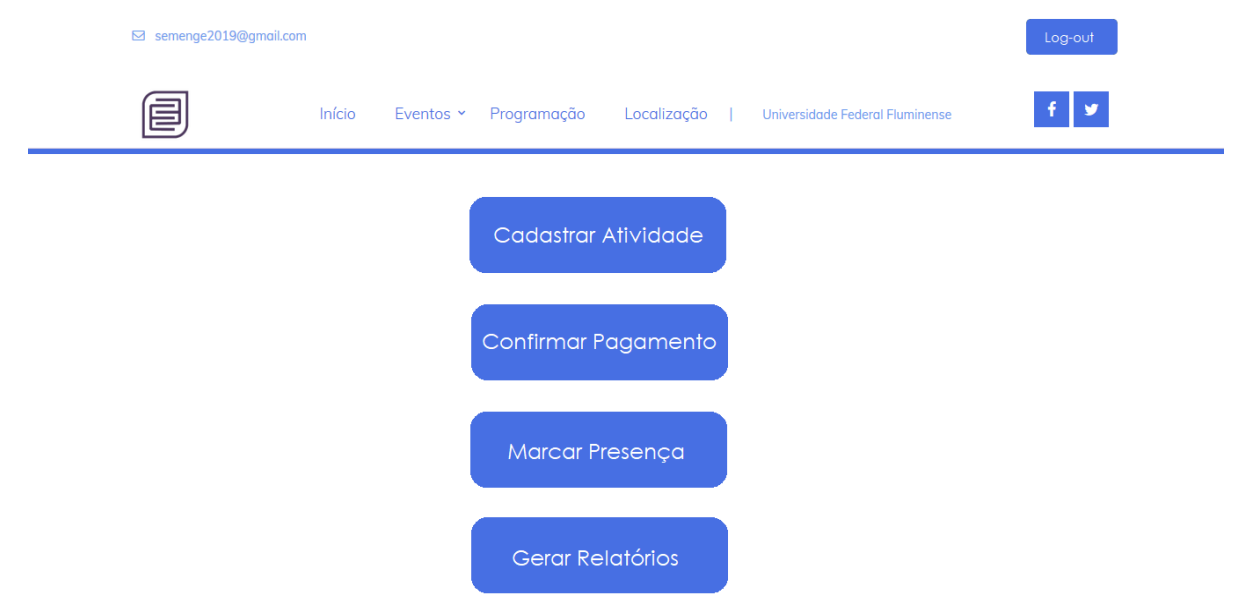

Figura 5 - Tela de ações de participante.

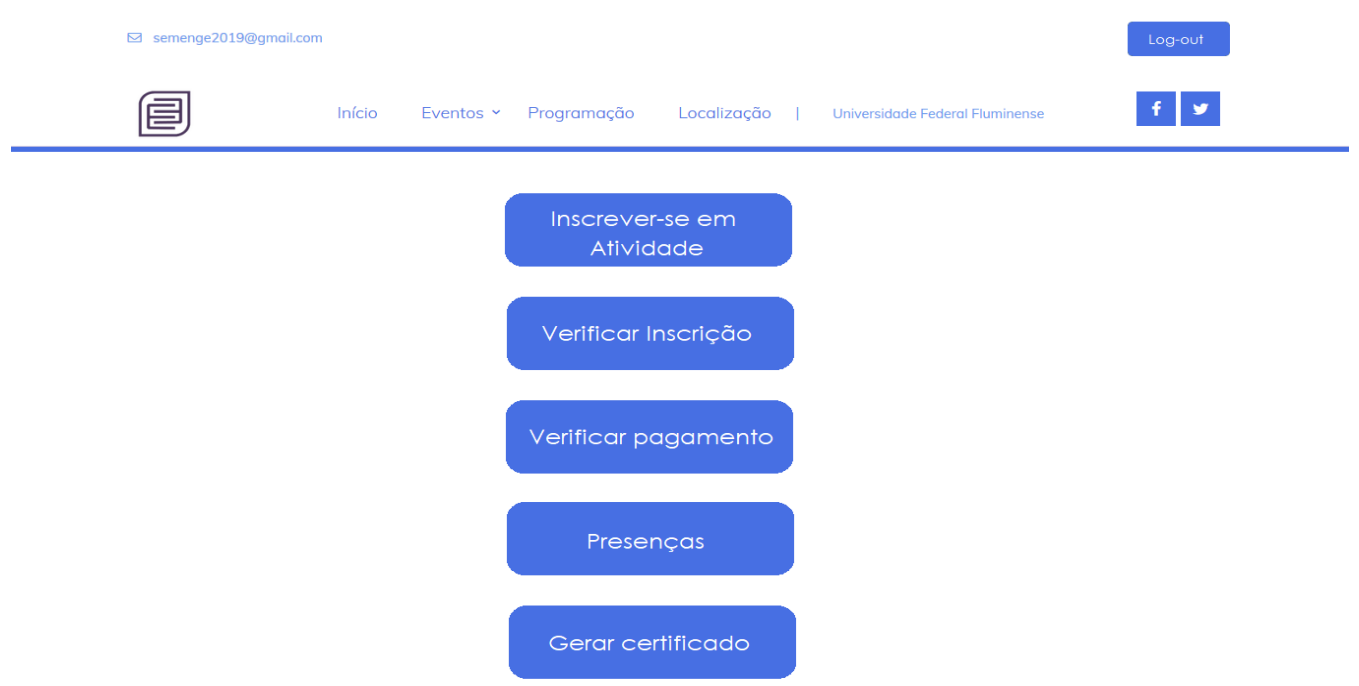




\section{CONSIDERAÇÕES FINAIS}

Em 2018, conhecendo o trabalho desenvolvido pelo PET-Tele, a Secretaria da Escola de Engenharia da UFF convidou o grupo a desenvolver, em caráter emergencial, um sistema de suporte à gestão da SEMENGE, evento anual organizado por tal Secretaria.

Aceitando o desafio, o PET-Tele levantou as necessidades, realizou um grupo de estudos, desenvolveu e implantou a primeira solução para um Sistema de Gestão, em uma rede interna da Secretaria (RANGEL JR. et al., 2019). O sistema foi utilizado na SEMENGE 2018. Durante o evento, manutenções foram realizadas e novas funcionalidades foram implantadas, a pedidos dos funcionários, que passaram a permitir uma análise dos dados do evento. A Secretaria emitiu um Voto de Louvor pelo trabalho do PET-Tele, em Boletim de Serviço da UFF, indicando o sucesso do sistema implantado (Boletim de Serviço UFF 1, 2020).

Em 2019, a Escola de Engenharia expandiu o evento, incluindo o Curso de Desenho Industrial e o renomeou para SEMENDE. Novamente, a Secretaria da Escola de Engenharia convidou o PET-Tele a desenvolver o Sistema de Gestão do evento. O PET-Tele novamente aceitou o desafio, desenvolveu e implantou uma nova solução para o Sistema de Gestão que foi usado no evento SEMENDE 2019.

Para o novo sistema, foram implantadas novas soluções, tanto para o Banco de Dados quanto para a Interface de Acesso Web. Embora a ideia de algumas tabelas tenha sido aproveitada da solução anterior, o novo Banco de Dados foi remodelado e novas tabelas foram inseridas. Em relação à interface $W e b$, novas webpages foram desenvolvidas, com características visuais e estruturais diferentes das originais, algumas delas com elementos responsivos.

A Secretaria emitiu um novo Voto de Louvor pelo trabalho do PET-Tele, em Boletim de Serviço da UFF, mais uma vez indicando o sucesso do novo sistema implantado (Boletim de Serviço UFF 2, 2020).

Como trabalhos futuros, podem-se destacar, pelo menos, os seguintes itens. A criação de webpages que apresentem informações mais detalhadas sobre o evento e instruções de navegação no sistema para os usuários. Além disso, em relação à segurança da informação, apesar de ter sido implantado um mecanismo de login, deve ser realizado um estudo mais aprofundado sobre o assunto, a fim de torná-lo mais robusto e de garantir uma maior proteção de dados de usuários. Do ponto de vista estrutural, pode-se sugerir ainda a utilização de um framework que facilite uma possível troca do SBGD empregado, caso ela se faça necessário. Por fim, recomenda-se que o sistema seja transferido das máquinas da secretaria da Escola de Engenharia para um servidor próprio (interno ou externamente contratado), a fim de proporcionar mais robustez à operação do sistema.

\section{Agradecimentos}

O grupo PET-Tele da UFF faz parte do Programa de Educação Tutorial (PET), financiado pelo Ministério da Educação (MEC).

O grupo PET-Tele agradece ao professor Carlos E. Pantoja (CEFET/RJ), por sempre se colocar gentilmente à disposição do grupo, ajudando a formar pessoal e profissionalmente diversos de nossos alunos.

O grupo PET-Tele agradece à Secretaria da Escola de Engenharia da UFF e aos seus funcionários, pelo convite, pela confiança, pelo trabalho em conjunto e por seus comentários e sugestões.

Os autores agradecem aos demais bolsistas do grupo PET-Tele, por ajudarem no desenvolvimento do projeto e na correção do presente artigo. 
(C) COBENGE

"Os desafios para formar hoje o engenheiro do amanhã"

\section{REFERÊNCIAS}

ACHOUR, M.; BETZ, F.; DOVGAL, A.. Manual de PHP.

Disponível em: <http://www.php.net/ >. Acesso em: 25 jun. 2020.

Boletim de Serviço UFF 2. BS-40- 09/03/2020 - Voto de Louvor para o Grupo PET-Tele.

Disponível em: <http://www.telecom.uff.br/pet/petws/downloads/homenagens/

BS_UFF_LIV_040_pg_071_Homenagem_PET_Tele_2020_03_09.pdf $>$.

Acesso em: 25 jun. 2020.

Boletim de Serviço UFF 1. BS-182- 09/10/2018 - Voto de Louvor para o Grupo PET-Tele.

Disponível em: <http://www.telecom.uff.br/pet/petws/downloads/homenagens/

BS_UFF_LII_182_pg_032_Homenagem_PET_Tele_2018_09_21.pdf $>$.

Acesso em: $2 \overline{5}$ jun. 2020 .

CSV-RFC. CSV RFC-4180. Disponível em: < https://tools.ietf.org/html/rfc4180>.

Acesso em: 25 jun. 2020.

CSV-WIKI. CSV Wikipedia.

Disponível em: < https://en.wikipedia.org/wiki/Comma-separated_values>.

Acesso em: 25 jun. 2020.

DINIZ, P. C.; FONTES, I. M. F. M.; PEIXOTO, J. A.; DE LA VEGA, A. S.. Desenvolvimento e implantação de um banco de dados para bibliografia de cursos de graduação. Anais: XLI Congresso Brasileiro de Educação em Engenharia - COBENGE. Gramado, RS, 2013.

EIS, DIEGO. Uma Breve História do CSS. Disponível em:

$<$ http://tableless.com.br/uma-breve-historia-do-css/> Acesso em: 25 jun. 2020.

LEWIS, J.; MOSCOVITZ, M.. CSS Avançado. Novatec, 2010. 416p.

MINISTÉRIO DA EDUCAÇÃO. Apresentação - PET. Disponível em:

$<$ http://portal.mec.gov.br/index.php?option $=$ com_content\&view $=$ article\&id $=12223$

\&ativo=481\&Itemid $=480>$. Acesso em: 25 jun. 2020.

MySQL. Linguagem de programação MySQL.

Disponível em: <http://www.mysql.com/>. Acesso em: 25 jun. 2020.

NIEDERST, J.. HTML: Pocked Reference. 2nd. edt. O’Reilly \& Associates, 2002. 33p.

OLIVEIRA, D. C.; WOYAMES, P. N.; PANTOJA, C. E.; DE LA VEGA, A. S.. Reestruturação e expansão de um banco de dados com acesso Web para bibliografia dos cursos de graduação em Engenharia da Universidade Federal Fluminense. Anais: XLIV Congresso Brasileiro de Educação em Engenharia - COBENGE. Natal, RN, 2016.

PACIEVITCH, Yuri. HTML. Disponível em:

$<$ http://www.infoescola.com/informatica/html/> Acesso em: 25 jun. 2020. 
C COBENCE 2020

"Os desafios para formar hoje o engenheiro do amanhã"

PDF. Portable Document Format.

Disponível em: $<$ http://acrobat.adobe.com/us/en/why-adobe/about-adobe-pdf.html $>$.

Acesso em: 25 jun. 2020.

PHP. Linguagem de programação PHP.

Disponível em: <http://secure.php.net/ >. Acesso em: 25 jun. 2020.

PET-TELE. PET - Engenharia de Telecomunicações da UFF.

Disponível em: <http://www.telecom.uff.br/pet>. Acesso em: 25 jun. 2020.

RANGEL JR., A. S.; ALMEIDA, B. M.; OLIVEIRA, F. B.; DE LA VEGA, A. S.. SISTEMA PARA GESTÃO DE EVENTO BASEADO EM BANCO DE DADOS COM INTERFACE WEB PARA A SECRETARIA DA ESCOLA DE ENGENHARIA DA UNIVERSIDADE FEDERAL FLUMINENSE. Anais: XLVII - Congresso Brasileiro de Educação em Engenharia - COBENGE. Fortaleza, CE, 2019.

RANGEL JR., A. S.; ALMEIDA, B. M.; OLIVEIRA, F. B.; PANTOJA, C. E.; DE LA VEGA, A. S.. IMPLANTAÇÃO DE UM BANCO DE DADOS COM ACESSO WEB PARA A COORDENAÇÃO DE ESTÁGIOS DA ESCOLA DE ENGENHARIA DA UNIVERSIDADE FEDERAL FLUMINENSE. Anais: XLV - Congresso Brasileiro de Educação em Engenharia - COBENGE. Joinville, SC, 2017.

SANTOS, B.S.; DE LA VEGA, A.S.. Desenvolvimento de Ferramenta de Auxílio Didático: Implementação de Interfaces com o Usuário via Internet usando CGILua. Anais: XL Congresso Brasileiro de Educação em Engenharia - COBENGE. Belém, 2012.

SILVA, M. CSS e Padrões Web.

Disponível em: < http://www.maujor.com/index.php> Acesso em: 25 jun. 2020.

SQL. Linguagem de programação SQL.

Disponível em: <http://www.w3schools.com/sql/>. Acesso em: 25 jun. 2020.

W3SCHOOLS. CSS Tutorial.

Disponível em:<http://www.w3schools.com/css/default.asp> Acesso em: 25 jun. 2020.

YOUNG, C. S. Banco de Dados: Organização, Sistemas e Administração. Universidade do Texas: Atlas, 1983. 398p. 


\title{
NEW SYSTEM FOR EVENT MANAGEMENT BASED ON DATABASE WITH WEB INTERFACE FOR THE SECRETARIAT OF THE SCHOOL OF ENGINEERING OF THE FEDERAL FLUMINENSE UNIVERSITY
}

\begin{abstract}
This paper presents a new system for the collaborative project carried out by the PET-Tele group for the Secretariat of the School of Engineering, at the Federal Fluminense University (UFF). The PET-Tele developed and deployed the first version of a Management System for the SEMENGE 2018 (Engineering Week) event, based on a Database, using SQL (Structured Query Language), with a Web interface, using HTML (HyperText Markup Language), PHP (Hypertext Processor) and CSS (Cascading Style Sheets). The basic motivation of the project was to meet an emergency demand from the Secretariat, which reported the loss of the previous system. The system proposed by PET-Tele came on stream in time to attend the event and was successfully used. For the 2019 version of the event, now called SEMENDE, the PET-Tele developed a new system, which is described in this paper.
\end{abstract}

Keywords: Tutorial Education Program (PET). Management system. Academic event. Database. Web access. 